\title{
LA FORMACIÓN DIGITAL EN LOS PROGRAMAS DE PERIODISMO DE EsPAÑA Y PORTUGAL
}

\author{
Pilar Sánchez-García* \\ Sandra Marinho**
}

\begin{abstract}
Resumen. Los cambios en el panorama de los medios de comunicación y el entorno digital y multimedia requieren una adaptación de los programas de enseñanza del periodismo. Esta investigación tiene como objetivo analizar cómo las universidades portuguesas y españolas han aprovechado el cambio de modelo educativo europeo del Plan Bolonia para reestructurar los planes de estudios, especialmente en relación con los contenidos tecnológicos y digitales. Los resultados reflejan mayor cantidad de asignaturas dedicadas a los contenidos digitales/ tecnológicos, con una amplia variedad de denominaciones, las cuales combinan contenidos tecnológicos genéricos junto a la práctica periodística multimedia. Estos resultados permiten desencadenar una discusión sobre la estrategia de la enseñanza del periodismo que incluya diversas opciones curriculares.
\end{abstract}

Palabras clave. Periodismo, enseñanza, Plan Bolonia, digital, multimedia.

\section{DIGITAL TRAINING IN JOURNALISM CURRICULA IN SPAIN AND PORTUGAL}

Abstract. Changes in the media landscape and the digital and multimedia environment require new directions in the way journalism is trained. This research aims to understand to what

* Profesora en la Universidad de Valladolid, España. Correo electrónico: pilar.sanchez@ hmca.uva.es

** Profesora en la Universidade do Minho, Portugal. Correo electrónico: marinho@ics. uminho.pt 
extent Portuguese and Spanish universities have used the implementation of the Bologna Declaration by European Union countries as an opportunity to restructure journalism curricula, especially regarding technological and digital contents. The results show an increasing number of courses devoted to digital/technological contents. Besides, there is a wide variety of denominations. These courses comprise more generic contents (related to digital and technological issues) but also subjects specifically devoted to journalism practice. These results allow us to trigger a discussion on the journalism training strategy that may be involved in these curricular options.

Key words. Journalism, education, Bologna Process, digital, multimedia.

\section{INTRODUCCIÓN}

El nuevo entorno mediático que transforma los medios de comunicación afecta directamente al debate sobre las nuevas necesidades formativas de los periodistas. La cuestión de cómo integrar la enseñanza tecnológica para un periodismo multimedia ha provocado cambios en los planes de estudio de esta titulación.

Resulta ya casi imposible separar la enseñanza de los cibermedios del periodismo tradicional, las barreras se difuminan para dirigirse hacia un periodismo multisoporte y deben entenderse las herramientas digitales como conaturales a cualquier actividad periodística (Salaverría, 2000). Su enseñanza se impone en las facultades de periodismo al tiempo que avanza la investigación sobre un fenómeno nuevo que ha transformado la comunicación de manera irreversible. La base tecnológica que está detrás del periodismo en las redes pone de relieve la vocación para la investigación aplicada y la innovación tecnológica, hasta este momento casi inexistente (López, 2010: 302). Investigación y enseñanza, en este caso, van más de la mano que nunca.

La nueva era digital propone un desafío formativo único en la titulación de periodismo que requiere corresponder a las expectativas de 
la sociedad que demanda profesionales competentes para las industrias mediáticas. Ésta es la batalla que deben afrontar los educadores del nuevo siglo (Marques de Melo, 2007).

El desafío formativo de la nueva sociedad de la información se articula en torno a tres cuestiones fundamentales: la integración del periodismo digital más allá de la técnica (Marinho, 2012), la formación de nuevos perfiles (Sánchez-García, Campos-Domínguez y Berrocal, 2015) y el cambio de mentalidad hacia la formación permanente y cambiante (López, 2010; Sánchez-García y Berrocal, 2014).

La transformación tecnológica global se ha unido, en el caso de Europa, a un cambio de modelo educativo de relevancia. La adaptación al Espacio Europeo de Educación Superior (EEES) ha unificado los criterios educativos de 47 países con la homologación de los títulos universitarios, con la vista puesta en favorecer una mayor movilidad laboral de los titulados europeos. En el caso de los estudios de periodismo, integrados en la rama de la comunicación, el cambio educativo coincide con la importante transformación tecnológica del sector. Por lo tanto, en los países implicados en el llamado Plan Bolonia, asistimos a una doble coyuntura o doble reto formativo: la adaptación de la convergencia educativa en Europa en un momento de profunda transformación del sector.

En este contexto se enmarca esta investigación que se centra en la realidad formativa de los periodistas de la península ibérica, dentro del área mediterránea. El estudio parte de un interrogante concreto: ¿las universidades de periodismo españolas y portuguesas han aprovechado el nuevo modelo educativo europeo para introducir los nuevos cambios formativos que demanda la era digital?

A partir de esta pregunta, el objetivo de esta investigación conjunta e internacional es realizar un análisis de los planes de estudio de periodismo que ofrecen las facultades españolas y portuguesas para comprobar la variación de su oferta formativa en nuevos medios antes y después de su adaptación al EeEs.

La hipótesis principal es que las facultades con estudios de periodismo de la península ibérica sí han aprovechado la adaptación a la convergencia educativa europea para incrementar la formación en torno al periodismo digital con un enfoque más práctico y técnico. 
MARCO TEÓRICO

La relación entre la formación y la tecnología digital ha estado presente en la literatura científica, por medio de la discusión de experiencias y adaptaciones curriculares ${ }^{1}$ cuando se debate sobre nuevas metodologías de enseñanza y la adaptación de estrategias formativas (List, 2001; Beam et al., 2003; Buckingham et al., 1999; Voakes et al., 2003).

Pavlik (2001) señala un enfoque más específico sobre los efectos en la enseñanza, identificando cinco grandes áreas en las que considera que las herramientas del periodismo se transforman:

1) recogida y transmisión de información; 2) almacenamiento de la información, indexación y recuperación, especialmente de contenido multimedia; 3) procesamiento, producción y edición; 4) distribución o publicación, y 5) presentación, visualización y acceso. Estas funciones están a menudo interconectadas e integradas en herramientas técnicas (2001: 193).

Para este autor, las implicaciones de estos cambios en el periodismo se asientan en cuatro escenarios: 1) la forma en que enseñamos e investigamos periodismo y comunicación, o como los formadores hacen su trabajo; 2) el contenido de lo que enseñamos; 3) la estructura de las escuelas y los departamentos de periodismo, así como de las universidades y otras instituciones de enseñanza superior, y 4) las relaciones entre los profesores de periodismo y una variedad de públicos, incluyendo estudiantes, patrocinadores, competidores y otros (Pavlik, 2001: 213). Para este autor, sin embargo, más que dominar la técnica se trata de dominar los nuevos lenguajes y los nuevos formatos:

Aunque las habilidades fundamentales necesarias para un periodista que trabaje en un nuevo medio sean una buena capacidad para comunicarse, escribir y editar, también es esencial

\footnotetext{
${ }^{1}$ Machado y Palacios, 2007; Knight, 2003; Bromley y Purdey, 1998; Cochie, 2008; Huang, 2008; Verweij, 2009; Huang et al., 2006; Deuze, 2001; Rutigliano, 2007; Corrêa y Corrêa, 2004; Bierhoff y Schmidt, 1997; Dennis et al., 2003
} 
comprender las capacidades y la estética de los nuevos medios. Esto incluye desarrollar una apreciación por la naturaleza interactiva de los medios digitales y la red y aprender a pensar en nuevas formas para una narrativa (storytelling) no lineal sino multilineal (2001: 213; traducción propia).

Huang et al. (2006) identifican cuatro grandes áreas de convergencia de los medios que afectan directamente a la forma en que los periodistas deben ser formados en las universidades: 1) la convergencia de contenidos; 2) la convergencia de formatos (o tecnológica); 3) la convergencia empresarial (corporate), y 4) la convergencia de roles (2006: 226-227). Para estos autores, es la convergencia de roles lo que tiene más impacto en la formación de los periodistas, ya que requiere de un conjunto más amplio de competencias, dentro de los límites del concepto (muy criticado) de los "one-journalist-fits-all-media" o el "backpack journalist" (2006: 228).

Dado el alcance y el impacto de estos cambios, Huang et al. consideran que es una "necesidad urgente" que la formación en periodismo trate estas cuestiones y no adopte "la estrategia de esperar para ver" (2006: 224) que, a largo plazo, supone una desventaja. Además defiende que el "pensamiento crítico" es el factor esencial:

Un currículo ideal debe equilibrar el peso de las disciplinas dirigidas a fomentar habilidades técnicas y las encaminadas al pensamiento crítico, inclinándose a estas últimas. El pensamiento crítico y las habilidades técnicas pueden ir de la mano en lugar de competir por el tiempo lectivo. Un currículo equilibrado puede ayudar mejor a los estudiantes a recopilar, producir, editar y distribuir noticias con calidad; a materializar sus ideas de una forma más profesional y más creativa; y a que sean más capaces de adaptarse al mercado, especialmente en un escenario de crisis económica (Huang et al., 2006: 255; traducción propia).

En este sentido establecen que los principales retos en la convergencia educativa del periodismo está en la adaptación a los nuevos soportes y a las nuevas técnicas redaccionales (Sánchez-García y Berrocal, 2013) que 
obligan a replantearse la formación periodística, más que nunca, de una manera permanente.

\section{Estrategias para una enseñanza equilibrada}

El aula asume aquí un papel clave como el primer lugar donde los estudiantes deben ser expuestos a la nueva tecnología propia del trabajo en un sistema de cross-media, y deben familiarizarse con ella, para que puedan ser más competentes cuando los alumnos desempeñen su labor futura. Es decir, "las disciplinas de tecnología no deben dominar la formación de los estudiantes de periodismo y no debe ser enseñado sólo por los intereses de la tecnología. La tecnología debe estar al servicio del objetivo de hacer buen periodismo" (Huang et al., 2006: 255). Otro aspecto esencial es el trabajo en equipo: colaboración y cooperación. De esta forma, se preparan para la convergencia en las redacciones. Estas estrategias implican, por supuesto, preparación tecnológica por parte de los profesores y apertura para soluciones como "la enseñanza en equipo", lo que, inevitablemente, implica inversión financiera por parte de las instituciones. Mark Deuze (2004) igualmente señala la necesidad de preparar a los estudiantes para la convergencia en las redacciones, que es mucho más que una cuestión técnica:

El periodista multimedia puede ser visto como un profesional que es competente y confiado al trabajar como un individuo en el contexto de una industria en convergencia -lo que implica flexibilidad al pensar y actuar sobre el proceso de mudanza y adaptación-y, al mismo tiempo, es una parte de un colectivo en el que varios, si no la mayoría, de sus colegas se sienten estresados, desconfiados e incluso amenazados por el continuo proceso de informatización y convergencia (2004: 149).

Es decir, es preciso aprender a comprender y lidiar con todo este contexto organizacional en proceso de cambio, que no se puede reducir a la formación tecnológica, y en el que "un enfoque en un sentido" 
nunca podrá funcionar. Por eso, el autor apunta cinco estrategias que las escuelas, los cursos, los profesores y los estudiantes pueden adoptar: 1) pensar dos veces antes de poner la tecnología y la técnica como los principios fundamentales de un plan de estudios en convergencia; 2) centrarse explícitamente en la comprensión de la lógica multimedia; 3) permitir que la convergencia sea debatida (por profesores, estudiantes, etcétera) porque, en la práctica, será esto lo que va a dar a la gente un sentido de participación en el proceso; 4) promover una actitud crítica en todas las formas de pensar-enseñar lo multimedia, y 5) dar mayor importancia a la calidad de la interacción entre periodistas, profesores y estudiantes de las (antiguas) áreas (radio, televisión, periódicos, agencias, revistas, medios on line, y también relaciones públicas, marketing y comunicación estratégica), ya que, más adelante en sus carreras, es muy posible que exista la expectativa de que no se vean como competidores, sino como colegas (Deuze, 2004: 149). Esta idea de una mayor comunicación ha sido referida anteriormente y parece ser recurrente entre los autores que abordan esta cuestión.

Cochie (2008) ya abordó los cambios curriculares en 411 escuelas de periodismo en Estados Unidos y llegó a la conclusión de que los planes de estudio están de hecho cambiando, especialmente en el sentido de la convergencia y la formación multimedia, lo que puede hacerse mediante una remodelación drástica o incrementando los elementos digitales de las asignaturas que ya existen. Los cambios dependen del apoyo de las instituciones y del propio cuerpo docente y, naturalmente, de los recursos financieros.

Sarachan (2011) se preocupó, precisamente con una vertiente específica, por la evaluación de las estrategias que se han impulsado para lograr la convergencia en los planes de formación en periodismo, teniendo en cuenta que los centros de enseñanza pueden beneficiarse de los conocimientos de las prácticas y de los resultados de unas y otras. Para ello realizó una investigación con 110 programas, lo que le permitió darse cuenta de las soluciones que existen, las opciones, herramientas y tendencias emergentes.

Las escuelas tienen diversas prioridades para proceder a la adaptación de sus planes de estudio: contenidos a enseñar, el nombre de las disciplinas, cómo evaluar a los estudiantes o cómo elegir softwares o 
sistemas operativos. El punto de partida es que hay, de hecho, variaciones entre los programas, un fenómeno con diversas explicaciones:

Para formar estudiantes para la industria, los departamentosescuelas de periodismo y comunicación luchan con la implementación de un plan de estudios de convergencia. La formación en la convergencia representa un cambio filosófico y pedagógico que combina las mejores prácticas profesionales con el conocimiento tecnológico, para ayudar a los estudiantes a aprender las competencias requeridas para la, cada vez más basada en internet, práctica de comunicación, del periodismo, el video, a las redes sociales. Sin embargo, debido a las limitaciones financieras, una industria variable y el escepticismo y el miedo a la tecnología de algunos profesores, los cursos varían en su progreso hacia la adaptación a la convergencia (Sarachan, 2011: 160).

Al igual que los autores anteriormente citados, Sarachan también advierte de los peligros de "tratar los nuevos medios como un camino separado", "ya que eso coloca a la tecnología como elemento definitorio del plan de estudios" (2011: 162). Por lo tanto, la tecnología puede ser implementada implicando diferentes grados de cambio en los planes de estudio: de forma estática, complementaria o reajustar. Es decir, el plan de estudios puede ser inalterado, pueden incrementarse las nuevas disciplinas y técnicas o que sea revisada la totalidad de su filosofía y estructura. Éstas son, una vez más, opciones influidas por los recursos financieros y el compromiso del equipo docente. La sugerencia es trabajar de una manera sustentada:

En lugar de complicar las cosas, para desarrollar un plan de estudios más allá de los recursos disponibles, puede ser más eficiente un esfuerzo por combinar tipos de medios [...] Un plan de estudios para la convergencia debería estar basado en la "interdisciplinariedad", en lugar de un enfoque multidisciplinar, ya que no es deseable hacer un programa demasiado amplio y pesado [...] La filosofía de la interdisciplinariedad —en la que los campos de estudio sean integrados por la identificación de los elementos 
comunes- proporciona un mapa para abordar las semejanzas y diferencias esenciales en la comunicación de masas (Sarachan, 2011: 162).

En esencia, revela la idea de que las herramientas específicas que se utilizan pueden no ser tan importantes como la capacidad de movernos entre ellas, por lo que, para este autor, las escuelas "tienen la responsabilidad de preparar a los alumnos para ser hábiles en su capacidad de aprender nuevas competencias tecnológicas que les van a ser requeridas en el lugar de trabajo" (Sarachan, 2011: 172).

Se trata, en definitiva, como hemos mostrado de varias formas a lo largo de este apartado, menos de aprender nuevas tecnologías y software, y más de aprender a pensar en una lógica multimedia y desarrollar la versatilidad suficiente para procurar soluciones en entornos en transformación. Al final, sigue siendo primordial lo que no puede olvidarse en la enseñanza del periodismo:

En el periodismo, por mucho que el mundo cambie, hay siempre cosas que nunca deberían cambiar, incluyendo el verificar los hechos rigurosamente; confiar en las fuentes conocidas y respetables; presentar los hechos de manera imparcial; formular preguntas difíciles; y se adhieran los estándares éticos más elevados. Pero algunas cosas tienen que cambiar, o cambiarán inevitablemente, para mejor o peor, cosas como las herramientas del periodista moderno (Pavlik, 2001: 188).

\section{Los perfiles periodísticos y formativos emergentes}

En esta idea del periodismo de siempre para un periodismo nuevo coinciden investigaciones que reflejan cómo en medio de esta transformación mediática "las funciones del periodista no varían ante los perfiles multimedia emergentes" (Sánchez-García, Campos-Domínguez y Berrocal, 2015). Precisamente, en el debate sobre la formación en nuevos medios entra en juego la perspectiva de los nuevos roles o perfiles periodísticos que se ha convertido en una importante línea 
de investigación (López, 2001, 2010; Meso et al. 2010; Biondi, Miró y Zapata, 2010). El periodista del siglo xxi es ya irremediablemente digital en todo el proceso informativo — búsqueda, confirmación, interpretación y difusión- y en este sentido resulta ineludible plantear la enseñanza integrada y multimedia, anteriormente referida. Los interrogantes siguen abiertos: ¿con qué perfil formamos ahora a los periodistas? ¿Debe ser un perfil polivalente o especializado? ¿Las redacciones reclaman periodistas multitarea?

Estas diferentes cuestiones encuentran, igualmente, relación con la enseñanza tecnológica, puesto que muchos de los nuevos perfiles y tareas que se encomiendan a los periodistas se basan en las nuevas herramientas y en las nuevas labores periodísticas. "Los periodistas han tenido que asumir tareas que tradicionalmente eran propias de otros profesionales, lo que les ha forzado a desarrollar un perfil más complejo y exigente" (Masip y Micó, 2009: 93). Son cambios asociados a la tecnología por la necesidad de afrontar la producción de contenidos multisoporte y pueden considerarse como una diversificación del mercado laboral (Mellado et al., 2007).

Los perfiles profesionales, a diferencia de las cualidades y las funciones, evolucionan de forma rápida con los cambios de la sociedad, parejos a las herramientas tecnológicas disponibles, la demanda de servicios nuevos y el mercado laboral del momento. La convergencia actual lo define como "renovados perfiles profesionales" (López, 2010: 232) que resultan dinámicos y cambiantes. De momento, la realidad profesional parece ir por delante de la realidad académica con la aparición de nuevos roles en redacciones, empresas, gabinetes y en la opción del trabajo freelance que aún no están incluidos en los programas educativos de manera integral, como trata de mostrar esta investigación.

En este contexto, es necesario hacer hincapié en la oportunidad y pertinencia de relacionar el cambio de perfiles profesionales con las nuevas necesidades educativas de los periodistas (Mellado et al., 2007; Balandrón, 2010; Sierra, 2011; López, 2012; Rosique, 2013), proponiendo un mayor desarrollo de la formación en nuevos roles periodísticos, cuestión aún en discusión que requiere de un esfuerzo de delimitación en el que confluyan las respuestas del ámbito académico y profesional. 
En este sentido, los roles emergentes, como se ha señalado, surgen como nuevas oportunidades laborares en las que el periodista puede trabajar "al servicio de empresas, instituciones, organizaciones, etcétera, para ponerlas en contacto con sus públicos interlocutores sociales, con y sin mediación de la prensa" (Piñuel, 2000: 5), por ejemplo. Meso et al. (2010) proponen los nuevos roles ajustados a tres grandes clasificaciones: periodista multimedia (maneja diferentes soportes), periodista multitarea (asume varias labores a la vez) y el periodista multiplataforma (difunde su trabajo por diferentes canales). Desde una perspectiva más utilitarista, Sánchez-García, Campos-Domínguez y Berrocal (2015) proponen cuatro nuevos perfiles formativos para incorporar a los planes de estudio: redactor multimedia polivalente o especializado; profesional de un gabinete de comunicación multifacético; profesor-investigador de laboratorio, y periodista promotor de servicios multimedia y community manager.

Se trata de propuestas abiertas en un debate inacabado en torno a la necesidad de reajustar la enseñanza periodística a las nuevas necesidades comunicativas y a un perfil profesional y formativo cambiante que debe seguir siendo objeto de investigación.

\section{Metodología}

En este contexto teórico y de partida, la presente investigación trata de conocer el perfil formativo de los periodistas del siglo xxi analizando la enseñanza que reciben en contenidos digitales, multimedia o de nuevas tecnologías. Para su estudio se recurre a una metodología de análisis de contenido cuantitativo y cualitativo que permite comparar los programas de periodismo que ofrecen los centros de enseñanza superior de España y Portugal antes y después de su adaptación a la convergencia educativa europea, con especial atención en las variaciones detectadas en las asignaturas obligatorias que se centran en la enseñanza de nuevos soportes y medios.

El campo de análisis se delimita desde dos perspectivas: la selección de los programas y las asignaturas a analizar. En el caso de España se incluyen los 31 planes antiguos de licenciatura (organizados en dos ciclos 
de cuatro a cinco años), extinguidos en 2015, y los 35 nuevos planes de grado en periodismo ( $1^{\circ}$ ciclo de cuatro años; no se tiene en cuenta el máster de $2^{\circ}$ ciclo) adaptados entre 2008 y 2010; y sin tener en cuenta otros grados en comunicación ni dobles grados que mezclan varias titulaciones. En el caso de Portugal, se incluyen los programas antiguos de licenciatura (28) y los nuevos planes de licenciatura, que mantienen su denominación, con 32 planes de estudio en total, implantados desde 2007 donde están incluidas universidades e institutos politécnicos.

En cuanto a las asignaturas objeto de observación, la investigación se centra en las materias obligatorias que conforman el perfil formativo básico de los estudiantes de periodismo y que permite conocer a qué contenidos se da prioridad o cuáles se consideran esenciales en los programas analizados. Por esta razón, no se tienen en cuenta en la cuantificación las asignaturas optativas o de libre configuración que dependen de la elección de cada alumno, aunque sí han formado parte de una observación general para comprender mejor la interpretación de algunos resultados concretos.

La selección de las asignaturas objeto de estudio se ha basado en su denominación, recogiendo una amplia variedad de términos que hacen referencia expresa a la enseñanza de las nuevas tecnologías, con acepciones como "tecnología de la información", "multimedia", "digital", "internet" y "ciberperiodismo", principalmente, y sin entrar en el contenido de las materias. Las dimensiones que se someten a estudio son cinco:

D.1. Número de asignaturas de "nuevas tecnologías" en los planes antiguos y nuevos.

D.2. La continuidad o variación de esta formación tecnológica en los programas.

D.3. La media del número de asignaturas de nuevas tecnologías en cada programa.

D.4. Su distribución por cursos.

D.5. La denominación de las materias.

Esta plantilla de análisis se ha aplicado a los planes de estudio de periodismo que se ofrecen en Portugal y en España. La lectura de resultados 
recoge, por un lado, las peculiaridades de cada país para posteriormente establecer un análisis comparativo conjunto.

En la aplicación del análisis existen limitaciones. Como se verá, se midieron y analizaron las mismas variables-dimensiones en los programas de ambos países, pero no siempre de la misma manera, usando exactamente las mismas medidas o indicadores. Por esta razón, no siempre los resultados para cada país son presentados del mismo modo, aunque esta circunstancia no invalida la comparación. Esta limitación se deriva del hecho de que se trata de investigaciones independientes, realizadas en diferentes fechas por diferentes investigadoras, que ahora se presentan relacionadas para establecer una comparativa general.

Otra limitación se refiere al hecho de que, incluso si estamos hablando del mismo fenómeno formativo - la adaptación al EeEs - se comparan periodos diferentes: mientras que en Portugal todo el proceso de transición al Plan Bolonia terminó en 2007 —a este año se refieren los datos presentados en esta investigación-, en España los datos se recogieron entre 2008 y 2010, fecha de implantación del Plan Bolonia. De esta forma, los resultados de esta investigación no pueden considerarse un reflejo inamovible de los "nuevos" planes de estudio en la actualidad, especialmente en el caso portugués, donde los planes han experimentado mayores modificaciones, y teniendo en cuenta, además, que España ya ha anunciado su intención de introducir nuevos cambios universitarios. Por ello, este análisis debe entenderse como una "foto fija" de la primera fase de adaptación al EEES de los estudios de periodismo en España y Portugal en cuanto a la enseñanza de los nuevos medios.

\section{DiSCUSIÓN DE LOS RESULTADOS}

En el caso de España, el estudio comparativo entre planes antiguos y nuevos (2010) muestra que en la primera dimensión del estudio (D1), $81 \%$ de los programas de grado en periodismo (25) cuenta con materias de nuevas tecnologías, mientras que 19\% (6) restante carece de esta oferta educativa en sus asignaturas obligatorias. En cambio, al 
pasar a la nueva titulación adaptada al Plan Bolonia, la oferta formativa en nuevas tecnologías está presente en $100 \%$ de los planes de estudio obligatorios de las 35 facultades con grado en periodismo. Esta misma variable aplicada a Portugal refleja que únicamente cuatro planes de estudio nuevos (2007) no ofrecen asignaturas obligatorias relacionadas con las "tecnologías" o "medios digitales" y todos los programas sí las ofrecen como asignaturas optativas.

La lectura de la variable de continuidad (D2) muestra que 55\% de las facultades en España aumenta el contenido relacionado con la formación en nuevas tecnologías (17), mientras que 22\% mantiene el mismo número de asignaturas obligatorias en ambas titulaciones (7) y $22 \%$ (7) reduce el número de asignaturas obligatorias relacionadas con nuevas tecnologías. ${ }^{2}$ Estos datos reflejan que la oferta en nuevas tecnologías está presente en la mayoría de las titulaciones con grado, pero el cambio de titulación no supone un incremento en número de asignaturas, porque $45 \%$ opta por mantener o reducir el número de materias obligatorias destinadas a la formación en nuevas tecnologías. En el caso portugués, en comparación con el periodo anterior a Bolonia (Fernandes, 2007) los centros de enseñanza públicos registraron un aumento del número de asignaturas, de 12 a 25 materias obligatorias (más del doble), y no se registran casos en los que haya disminuido el número de asignaturas.

La dimensión referida a la media de asignaturas de nuevas tecnologías en el conjunto de los programas (D3) evidencia que en el caso de España los planes antiguos ofrecían una horquilla de entre una y dos asignaturas obligatorias en 58\% de los programas. Este valor aumenta al cambiar a los nuevos planes con un número mayoritario de entre dos y tres asignaturas relacionadas con las nuevas tecnologías en $80 \%$ de los programas. Un dato que contextualiza al tenerse en cuenta que el número de asignaturas obligatorias de cada nuevo programa en España oscila entre 35 y 40 materias en los cuatro años, es decir, que de ese total de materias obligatorias, entre dos y tres se centran en nuevas tecnologías, de media. En Portugal, después de Bolonia (2007) existen

\footnotetext{
2 Esta reducción de materias se explica porque algunas facultades optan por convertirlas en optativas.
} 
programas que solamente ofrecen una asignatura obligatoria de nuevas tecnologías, frente a los que ofrecen cinco o seis, o los que limitan a dos asignaturas (nueve programas). Además, en Portugal, el número de disciplinas obligatorias en los planes de estudio varía entre 30 y 36. Si tenemos en cuenta que, en total, hay 70 asignaturas obligatorias y 18 opcionales en 32 planes de estudio, se puede decir que hay una media de 2.2 asignaturas obligatorias relacionadas con las nuevas tecnologías por cada plan de estudios.

El análisis de la distribución por cursos (D4) refleja que tanto en los planes nuevos como en los antiguos de periodismo en España, el grueso de las materias relacionadas con nuevas tecnologías predomina en $3^{\circ}$ y $4^{\circ}$ curso. Es decir, hay una tendencia a la especialización multimedia, digital o tecnológica en la última etapa de la titulación. En el caso portugués, podemos ver una doble tendencia: hay asignaturas de nuevas tecnologías de formación general en los primeros curso y asignaturas específicas de periodismo al final, lo que demuestra, como en España, una tendencia hacia la especialización al final del curso pero también una preocupación por proporcionar a los estudiantes las herramientas digitales al inicio de su formación.

Por último, en cuanto a las diferencias en la denominación de las materias (D5), la diversidad es tan amplia que se ha optado por dividir esta variable en dos unidades de análisis, al agrupar, por un lado, las materias cuya denominación se refiere de forma genérica a "tecnologías de la información" (con variedades como tecnología de la comunicación, fundamentos de la tecnología, nuevas tecnologías aplicadas al periodismo, etcétera) y las denominaciones específicas (que agrupan variedades como ciberperiodismo, digital, electrónica, cibernético, internet, red, interactiva, etcétera).

Los datos muestran que en los planes antiguos españoles abundan las denominaciones genéricas referidas a "tecnologías" (58\%) frente a las específicas referidas a nuevos soportes, multimedia o digital (41\%). Un resultado que experimenta un cambio de tendencia en el nuevo plan de estudios con la preponderancia de materias con denominación específica $(76 \%)$ frente a las genéricas (24\%). En el caso de las denominaciones específicas, en las dos titulaciones predominan tres acepciones: electrónico, digital y multimedia. 
En cuanto a la denominación de estas materias en Portugal, en los planes anteriores a Bolonia se confirma la existencia de dos grandes grupos de asignaturas: uno, relativo a las tecnologías en general, y otro, específicamente asociado al periodismo; y, de hecho, el término "digital" se emplea solamente en la denominación de una única asignatura. Con la nueva titulación, se perciben dos cambios semánticos en relación con el periodo anterior: el término "digital" es usado con más frecuencia en los planes nuevos y surge el término "multimedia". Además, se detectan los mismos dos grupos de asignaturas: las que se denominan como "tecnologías", "digital" y "multimedia" en términos generales y las que se centran en periodismo ("ciberperiodismo", "periodismo digital", "periodismo web" o "periodismo multimedia").

\section{Conclusiones}

La investigación evidencia que existe un incremento de asignaturas obligatorias relacionadas con la enseñanza de las nuevas tecnologías al cambiar de los planes antiguos a los nuevos en los dos países, aunque en mayor proporción en el caso de Portugal que en el de España.

El cambio de modelo educativo de la enseñanza superior con la adaptación a la convergencia educativa europea ha sido aprovechado para introducir las novedades tecnológicas del sector, aunque en España de una manera limitada, dejando la mayor parte de esta oferta digital en los itinerarios optativos que dependen de los alumnos. La explicación de que el cambio es mayor en Portugal desde 2007 se encuentra en el hecho de que la adaptación al Plan Bolonia, reduciendo los estudios a tres años, se entendió como una oportunidad para cambiar los planes de estudio en profundidad y actualizarlos, de manera que en este momento, en Portugal, existe una gran apuesta por las nuevas tecnologías en la educación en general. Esta situación contrasta con la de España, cuyo modelo educativo realizó una adaptación más moderada al Plan Bolonia en 2010, manteniendo cuatro años de titulación con una importante continuidad de sus planes de estudios, circunstancia que puede cambiar a medio plazo, puesto que el gobierno español ha anunciado ya una segunda fase de convergencia educativa europea, 
recortando la titulación a tres años como en el caso de Portugal, lo que permite aventurar que su oferta aumentará la carga formativo en nuevos medios.

En los programas de estudio de ambos países se detecta una amplia variedad de denominaciones que abarcan desde las más genéricas (referidas a tecnologías) a las materias más especializadas (multimedia, digital, ciberperiodismo). Estas últimas aumentan en el caso de los nuevos planes de estudio en los dos país, demostrando que la formación de los periodistas incluye ahora mayor especialización en contenidos propios de la nueva era digital. Este tipo de enseñanza técnica y especializada refleja una clara tendencia de cambio al incrementar la carga práctica de los planes de estudio de periodismo, lo que ha implicado un recorte de contenidos teóricos propios del área de la comunicación y, especialmente, de otras áreas de las ciencias sociales y las humanidades.

En definitiva, las tendencias formativas de los periodistas en la península ibérica muestran que la formación en nuevos medios diluye la frontera entre los diferentes soportes tradicionales y multimedia, de manera que la enseñanza periodística del futuro requerirá una concepción multisoporte que deje atrás las formación específica y diferenciada entre prensa, radio, TV y medios digitales.

El debate expuesto establece la necesidad de organizar una nueva estrategia en la formación digital o multimedia de los periodistas, en la que se debe decidir entre visiones dicotómicas sobre la formación: por medios o multimedia; basada en la tecnología o basada en estudios de periodismo; centrada en asignaturas específicas o digital, o bien promover un entorno digital y transversal.

Desde esta investigación se resuelve que en la integración de las nuevas tecnologías conviene apostar por una formación multimedia e integral del periodista atendiendo a la idea de que el aprendizaje tecnológico es únicamente instrumental y que el periodista debe realizar un trabajo informativo, interpretativo y de orientación con criterios multimedia, más allá de la técnica. Por ello, la formación digital, que requiere integrar nuevos perfiles periodísticos especializados o polivalente, debe considerarse necesariamente transversal e incorporar aspectos teóricos y de análisis como resultado de una investigación científica del entorno multimedia que está dando sus frutos en el ámbito internacional. 
Esta investigación no se da aquí por cerrada, sino que puede considerarse como un punto de partida en un momento de transición mediática y educativa, por lo que abre la puerta a futuras investigaciones en torno a diferentes aspectos, como la evolución de la formación multimedia en los planes de estudio de periodismo en los próximos años y a la realización de análisis comparativos con otros países a medio y largo plazo.

\section{FuENTES CONSULTADAS}

Alves, A. (1995), "The Future of Communication Sciences in Portugal", en Sigma (ed.), The Future of Communication Sciences in Europe, Siena: Sigma, pp. 150-176.

Balandrón, A. (2010), "La docencia en Comunicación en el eeEs ante los perfiles profesionales emergentes: el caso de los community managers", en F. Sierra y F. Cabezuelo (coords.), Competencias y perfiles profesionales en los estudios de Ciencias de la Comunicación, Madrid: Fragua, pp. 206-219.

BeAm, R. A. et al. (2003), "Technology-induced Stressors, Job Satisfaction and Workplace Exhaustion among Journalism and Mass Communication Faculty", en Journalism \& Mass Communication Educator, vol. 57, núm. 4, Londres: Sage, pp. 335-351.

Bierhoff, J.; Schmidt, M. (eds.) (1997), European Journalism Training in Transition, Maastricht: European Journalism Centre.

Biondi, J.; Miró, S.; Zapata, E. (2010), Derribando muros. Periodismo 3.0: oferta y demanda de comunicación en el Perú de hoy, Lima: El Comercio.

Bromley, M.; Purdey, H. (1998), "Journo-Morphosis: Today's New Media and the Education and Training of Tomorrow's 'Cool' Journalists", en Convergence: The International Journal of Research into New Media Technologies, vol. 4, núm. 4, Londres: Sage, pp. 77-93.

Buckingham, D. et al. (1999), "The Difference is Digital? Digital Technology and Student Media Production", en Convergence: the International Journal of Research into New Media Technologies, vol. 5, núm. 4, Londres: Sage, pp. 10-24. 
Cochie, M. (2008), "A Curriculum Evolution: How Journalism Programs are Dealing with Convergence", en 2008 AEJMC Convention, Chicago (Illinois), 6-9 de agosto.

CorrêA, E. S.; CorrêA, H. L. (2004), "O ensino da comunicação e do jornalismo no panorama das mídias digitais: perspectivas para uma renovação do perfil de habilidades e competências", en V Congreso Iberoamericano de Periodismo en Internet, Brasil: Universidad Federal de Bahia, 25 de noviembre de 2004.

Dennis, E. et al. (2003), "Learning Reconsidered: Education in the Digital Age", Journalism \& Mass Communication Educator, vol. 57, núm. 4, Londres: Sage, p. 317.

Deuze, M. (2001), "Educating 'New' Journalists: Challenges to the Curriculum", en Journalism \& Mass Communication Educator, vol. 56, núm. 1, Londres: Sage, pp. 4-17.

Deuze, M. (2004), "What is Multimedia Journalism?", en Journalism Studies, vol. 5, núm. 2, Oxford: Taylor \& Francis, pp. 139-152.

Díaz-Noci, J. (2007), "Perspectivas de la investigación y docencia universitarias de la comunicación periodística", en B. Gómez y S. Hernández (eds.), Estudios de Periodística XIII. La Periodística como disciplina universitaria: balance y perspectivas, Pamplona: Diario de Navarra / Sociedad Española de Periodística, pp. 31 39.

FERnANDES, L. (2007), Da Universidade às Redacções - caminhos para um melhor ensino público de Jornalismo (Dissertação de Mestrado), Braga: Centro de Estudos de Comunicação e Sociedade-Universidade do Minho.

Huang, E. (2008), "Teaching Button-pushing vs. Teaching Thinking The State of New Media Education in U.S. Universities", 2008 AEJMC Convention, Chicago (Illinois), 6-9 de agosto.

Huang, E. et al. (2006), "Bridging Newsrooms and Classrooms: Preparing the Next Generation of Journalists for Converged Media", en Journalism \& Communication Monographs, vol. 8, núm. 3, Londres: Sage, pp. 221-262.

Knight, A. (2003), "Online Journalism Education: Reaching and Teaching Globalised Media”, en ejournalist, vol. 3, núm. 2, pp. $1-11$. 
List, K. (2001), "High-tech vs High-touch Teachers", en Journalism \& Mass Communication Educator, vol. 56, núm. 3, Londres: Sage, pp. 84-89.

López, X. (2001), "Nuevos perfiles de los periodistas en la sociedad de la información", en Ámbitos, núms. 7-8, Sevilla: Universidad de Sevilla, pp. 7-18.

López, X. (2010), "La formación de los periodistas en el siglo xxi en Brasil, España, Portugal y Puerto Rico", en Revista Latina de Comunicación Social, núm. 65, La Laguna (Tenerife): Universidad de La Laguna, pp. 231-243.

LóPez, X. (2012), "La formación de los periodistas para los entornos digitales actuales", en Revista de Comunicación, núm. 11, Lima: Facultad de Comunicación de la Universidad de Piura, pp. 178-195.

Machado, E.; Palacios, M. (2007), O Ensino do Jornalismo em Redes de Alta Velocidade, Salvador da Bahia: Editora da Universidade Federal de Bahia (Edufba).

Marinho, S. (2012), Formação em jornalismo numa sociedade em mudança: modelos, percepções e práticas na análise do caso português. Tesis de doctorado, Braga: Universidade do Minho-Instituto de Ciências Sociais.

Marques de Melo, J. (2007), "A batalha da qualidade no ensino de comunicaçâo: novos (antigos) desafios", en Margarida Kunsch (coord.), Ensino de Comunicaçâo. Qualidade na formação acadêmico-profissional, São Paulo: Escola de Comunicações e Artes-Universidade de São Paulo (ECA-USP) / Intercom, pp. 33-41.

Masip, P.; Micó, J. L. (2009), "El periodista polivalente en el marco de la convergencia empresarial", en Quaderns del CAC, núms. 31-32, Cataluña: Consejo del Audiovisual de Cataluña (CAC), pp. 91-99. Mellado, C. et al. (2007), "Investigación de perfiles profesionales en periodismo y comunicación para una actualización curricular permanente", en Zer, núm. 23, Vizcaya: Universidad del País Vasco, pp. 139-164.

Meso, K. (2003), "La formación del periodista digital", en Chasqui, Revista Latinoamericana de Comunicación, núm. 84, Quito: Quipus-Ciespal, pp. 4-11. 
Meso, K. et al. (2010), "Presencia y uso de internet en las redacciones de los diarios vascos y navarros", en Mediatika, núm. 12, Donostia: Sociedad de Estudios Vascos, pp. 301-319.

Montiel, M.; Villalobos, F. (2005), "La enseñanza del periodismo en el siglo xxi: un desafío entre lo impreso y lo digital", en Telos, vol. 7, núm. 3, septiembre-diciembre, Madrid: Fundación Telefónica, pp. 397-411.

Pavlik, J. V. (2001), Journalism and New Media, New York: Columbia University Press.

Pavlik, J. V. (2005), "Running the Technological Gauntlet: Journalism and New Media", en Hugo de Burgh (ed.), Making Journalists, Nueva York: Routledge, pp. 245-263.

Piñuel, J. L. (2000), "Formación universitaria y formación de profesionales en Métodos y Técnicas de Investigación social en Comunicación", en Congreso Nacional de Investigadores de la Comunicación Audiovisual. Artículo disponible en [http://web.jet.es/pinuel. raigada/], 12 de diciembre de 2012.

Rosique, G (2013), "Los estudios universitarios de periodismo en España: la adaptación al EEES y la formación de periodistas en competencias digitales", en Revista Internacional de Estudios sobre Sistemas Educativos, vol. 1, núms. 1-2, México: Universidad del Desarrollo Empresarial y Pedagógico, pp. 117-132.

Rutigliano, L. (2007), "The Future of Journalism and How to Teach It", en 8th International Symposium on Online Journalism, University of Texas-Austin, 30-31 de marzo.

Salaverría, R. (2000), "Criterios para la formación de periodistas en la era digital", en I Congreso Nacional de Periodismo Digital. Huesca, 14-15 de enero. Artículo disponible en [http://www.unav.es/ fcom/mmlab/mmlab/investig/crite.htm], 17 de febrero de 2014.

Salaverría, R. (2011), "Online Journalism Meets the University: Ideas for Teaching and Research", en Brazilian Journalism Research, vol. 7, núm. 11, Brasil: Universidade de Brasília, pp. 137-152.

Sánchez-García, P. (2013), "Desafíos en la formación de los periodistas españoles: convergencia europea, capacitación tecnológica y formación permanente", en Comunicación y Medios, núm. 28, Santiago: Universidad de Chile, pp. 40-60. 
Sánchez-García, P. (2014), Un siglo de enseñanza periodística en España: de la primera Escuela de Periodismo a la adaptación de los estudios al Espacio Europeo de Educación Superior. Tesis de doctorado, Valladolid: Facultad de Filosofía y Letras-Universidad de Valladolid.

Sánchez García, P.; Berrocal, S. (2014), "Desafíos de la enseñanza periodística en el EEEs: adaptación a los nuevos soportes y a las nuevas técnicas redaccionales", en E. Bernard (coord.), Actualización de los sistemas educativos, Madrid: Visión Libros, pp. 231-243.

Sánchez-García, P., Campos-Domínguez, E.; Berrocal, S. (2015), "Las funciones inalterables del periodista ante los perfiles multimedia emergentes", en Revista Latina de Comunicación Social, núm. 70, La Laguna (Tenerife): Universidad de La Laguna, pp. 187208.

Sarachan, J. (2011), "The Path Already Taken: Technological and Pedagogical Practices in Convergence Education", en Journalism \& Mass Communication Educator, vol. 66, núm. 2, Londres: Sage, pp. 160-174.

SierRa, F. (2011), "La formación científica del informador. Universidad y campo periodístico", en F. Sierra y F. J. Moreno (eds.), Fundamentos de Teoría del Periodismo, Sevilla: Universidad de Sevilla, pp. 11-39.

Tejedor, S. (2008), "Ciberperiodismo y universidad: diagnósticos y retos de la enseñanza del periodismo on-line", en Anàlisi, núm. 35. Barcelona: Universidad Autónoma de Barcelona / Universidad Oberta de Cataluña, pp. 25-39.

Tejedor, S. (2007), "La enseñanza del ciberperiodismo: enseñar y aprender periodismo on-line", en B. Gómez y S. Hernández (eds.), Estudios de Periodística XIII. La Periodística como disciplina universitaria: balance y perspectivas, Pamplona: Diario de Navarra / Sociedad Española de Periodística, pp. 405-411.

Tejedor, S. (2006), La enseñanza del ciberperiodismo en las licenciaturas de periodismo en España. Tesis de doctorado, Barcelona: Universidad Autónoma de Barcelona. Documento disponible en [http://www.tdx.cat/bitstream/handle/10803/4188/stcldel. pdf?sequence=1], 17 de junio de 2015. 
Verweij, P. (2009), "Making Convergence Work in the Newsroom: A Case Study of Convergence of Print, Radio, Television and Online Newsrooms at the African Media Matrix in South Africa During the National Arts Festival", Convergence: The International Journal of Research into New Media Technologies, vol. 15, núm. 1, London: Sage. pp. 75-87.

VoAkes, P. S. et al. (2003), "The Impact of Technological Change on Journalism Education: A Survey of Faculty and Administrators", Journalism \& Mass Communication Educator, vol. 57, núm. 4, Londres: Sage, pp. 318-335.

Fecha de recepción: 10 de agosto de 2015 Fecha de aprobación: 20 de abril de 2016 